Research Paper

\title{
Preliminary Results about Application of Intensity-Modulated Radiotherapy to Reduce Prophylactic Radiation Dose in Limited-Stage Small Cell Lung Cancer
}

\begin{abstract}
Zhiyan Liu, Jing Wang, Zhiyong Yuan, Baozhong Zhang, Linlin Gong, Lujun Zhao ${ }^{凶}$, and Ping Wang ${ }^{凶}$
Department of Radiotherapy, Tianjin Medical University Cancer Institute and Hospital, National Clinical Research Center for Cancer, and Key Laboratory of Cancer Prevention and Therapy, Tianjin's Clinical Research Center for Cancer, Tianjin 300060, P.R. China.

Co-first Author: Jing Wang

$\triangle$ Corresponding authors: Prof. Ping Wang, Tianjin Medical University Cancer Institute and Hospital, National Clinical Research Center for Cancer, Department of Radiotherapy and Key Laboratory of Cancer Prevention and Therapy, Tianjin's Clinical Research Center for Cancer, Tianjin 300060, P.R. China. Tel: (+86)022-23519953; Fax: (+86) 022-23341405; Email: wangping@tjmuch.com and Prof. Lujun Zhao, Tianjin Medical University Cancer Institute and Hospital, National Clinical Research Center for Cancer, Department of Radiotherapy and Key Laboratory of Cancer Prevention and Therapy, Tianjin's Clinical Research Center for Cancer, Tianjin 300060, P.R. China. Tel: (+86)022-23340123-6504; Fax: (+86) 022-23341405; Email: tjdoctorzhao@126.com

(c) Ivyspring International Publisher. This is an open access article distributed under the terms of the Creative Commons Attribution (CC BY-NC) license (https://creativecommons.org/licenses/by-nc/4.0/). See http://ivyspring.com/terms for full terms and conditions.
\end{abstract}

Received: 2018.01.16; Accepted: 2018.04.14; Published: 2018.06.23

\begin{abstract}
To evaluate the clinical outcomes of simultaneous dose reduction of elective nodal area with intensity-modulated radiotherapy (SIR-IMRT) versus conventional IMRT (C-IMRT) in patients with limited-stage small-cell lung cancer (LS-SCLC), patients with LS-SCLC who received definitive SIR-IMRT or C-IMRT were retrospectively analyzed. In SIR-IMRT group, the prescribed dose was $60 \mathrm{~Gy}$ to the planning gross target volume (PTV $\mathrm{F}_{\mathrm{G}}$ ), and 54Gy to the planning target volume (PTV). In the C-IMRT group, the prescribed dose was 60Gy to the whole PTV. Radiation-related toxicities were estimated according to Common Terminology Criteria for Adverse Events (version 3.0). The primary endpoint was loco-regional recurrence-free survival (LRFS), and the secondary endpoints include overall survival (OS) and toxicities. LRFS and OS were estimated with Kaplan-Meier method. After propensity score matching, 84 patients were included in this study, with 42 patients in each group. Fifty-eight patients experienced treatment failure. Grade 3 and above radiation-induced lung toxicity developed in 2 patients in SIR-IMRT group and 5 patients in C-IMRT group, respectively. Grade 3 and above radiation-related esophagitis was observed in 3 patients and 5 patients, respectively. The 1-, 3-year LRFS rates of the SIR-IMRT group and C-IMRT group were $90.4 \%$, $73.3 \%$ and $87.1 \%, 45.0 \%(P=0.025)$, respectively. Reducing the irradiation dose to the elective nodal region may reduce radiation-induced toxicities without compromising local-regional control and overall survival.
\end{abstract}

Key words: Small-cell lung cancer, Radiation therapy, Intensity-modulated radiotherapy

\section{Introduction}

Combining chemotherapy and thoracic radiotherapy (RT) has been shown to improve patient survival ${ }^{1,2}$ as the standard treatment for limited-stage small cell lung cancer (LS-SCLC). However, maintenance of local control is not reliably achieved for patients with $\operatorname{SCLC}^{3}$, so we need to find a better way to improve the local control without increasing radiation-induced toxicities. In the past several decades, increasing the strength of treatment, including radiation dose and patterns of radiation have been varied to optimize treatment to improve local control rate and prolong the survival period ${ }^{4-7}$. 
However, the results of a Radiation Therapy Oncology Group (RTOG)-0617 study of non-small cell lung cancer (NSCLC) were disappointing; the survival time of patients receiving 74Gy irradiation was less than 60Gy group. The reason may be associated with an increased treatment-related death in the high dose groups ${ }^{8}$. Thus, research is ongoing to optimize radiation delivery to tumors while sparing surrounding normal structures. The dose for controlling subclinical lesions compared with gross target volume is lower, and it is reported that the dosage of 50Gy can effectively control the subclinical lesions ${ }^{9}$.We were interested in simultaneous integrated dosed reduction to elective nodal area with intensity - modulated radiotherapy (SIR-IMRT). In this paper, patients with LS-SCLC accepting SIR-IMRT treatment or conventional IMRT (C-IMRT) treatment were retrospectively analyzed to evaluate the clinical effect of the two treatments.

Table 1. Patient characteristics $(n=84)$

\begin{tabular}{|c|c|c|c|}
\hline Characteristics & SIR-IMRT No.(\%) & C-IMRT No.(\%) & $P$ \\
\hline \multicolumn{4}{|l|}{ Age (y) } \\
\hline$<60$ & $27(64.3 \%)$ & $28(66.7 \%)$ & \multirow[t]{2}{*}{0.818} \\
\hline$\geq 60$ & $15(35.7 \%)$ & $14(33.3 \%)$ & \\
\hline \multicolumn{4}{|l|}{ Site } \\
\hline Left lung & $21(50.0 \%)$ & $20(47.6 \%)$ & \multirow[t]{2}{*}{0.665} \\
\hline Right lung & $21(50.0 \%)$ & $22(52.4 \%)$ & \\
\hline \multicolumn{4}{|l|}{ Type } \\
\hline Peripheral & $3(7.1 \%)$ & $2(4.8 \%)$ & \multirow[t]{2}{*}{0.951} \\
\hline Central & $39(92.9 \%)$ & $40(95.2 \%)$ & \\
\hline \multicolumn{4}{|l|}{ Clinical T stage } \\
\hline $\mathrm{T} 1$ & $5(11.9 \%)$ & $7(16.7 \%)$ & \multirow[t]{4}{*}{0.770} \\
\hline $\mathrm{T} 2$ & $25(59.5 \%)$ & $20(47.6 \%)$ & \\
\hline $\mathrm{T} 3$ & $7(16.7 \%)$ & $8(19.0 \%)$ & \\
\hline $\mathrm{T} 4$ & $5(11.9 \%)$ & $7(16.7 \%)$ & \\
\hline \multicolumn{4}{|l|}{ Clinical N stage } \\
\hline No & $2(4.8 \%)$ & $4(9.5 \%)$ & \multirow[t]{4}{*}{0.459} \\
\hline N1 & $3(7.1 \%)$ & $1(2.4 \%)$ & \\
\hline N2 & $26(61.9 \%)$ & $22(52.4 \%)$ & \\
\hline N3 & $11(26.2 \%)$ & $15(35.7 \%)$ & \\
\hline \multicolumn{4}{|l|}{ Clinical stage } \\
\hline $\mathrm{Ib}$ & $1(2.4 \%)$ & $2(4.8 \%)$ & \multirow[t]{5}{*}{0.394} \\
\hline IIa & $1(2.4 \%)$ & $2(4.8 \%)$ & \\
\hline $\mathrm{IIb}$ & $3(7.1 \%)$ & 0 & \\
\hline IIIa & $22(52.4 \%)$ & $19(45.2 \%)$ & \\
\hline IIIlb & $15(35.7 \%)$ & $19(45.2 \%)$ & \\
\hline \multicolumn{4}{|l|}{$\begin{array}{l}\text { Pathological } \\
\text { subtype }\end{array}$} \\
\hline Simple & $34(81.0 \%)$ & $34(81.0 \%)$ & 1.0 \\
\hline Composite & $8(19.0 \%)$ & $8(19.0 \%)$ & \\
\hline
\end{tabular}

\section{Material and methods}

Patients Patients with LS-SCLC underwent radical SIB-IMRT and C-IMRT from Jan. 2010 to Dec. 2013 at our hospital were collected, including 72 patients in SIR-IMRT group and 65 patients in C-IMRT group. Propensity score matching (PSM) was performed based on the factors including gender, age, stage of disease, and chemotherapy, and after matching, a total of 84 patients were included in this study, with 42 patients in each group. The pathologic diagnoses of SCLC of all the patients were confirmed by two senior pathologists. The median age was 58 years old (range,38-74). Sixty-one patients were males and 23 were females, the ratio was 2.65:1. Panofsky Performance Status (KPS) score of all patients were no less than 70. All patients received Serologic examinations including blood routine and hepatic and renal function tests, chest computed tomography (CT), neck and abdomen ultrasound, brain magnetic resonance imaging (MRI), and bone scan imaging before radiotherapy. Clinical stage was conducted by the imaging examination according to the current American Joint Committee on Cancer (AJCC) criteria for NSCLC. The general information of patients was shown in Table 1.

Therapy All Patients underwent positioning scan by large-aperture spiral CT simulator. The slice thickness was $5 \mathrm{~mm}$. The gross tumor volume (GTV) was defined as any visible primary tumor and lymph nodes on CT simulation. All lymph nodes were conducted as positive lesions if they met one or more of the following conditions: with a short diameter $\geq 10 \mathrm{~mm}$, central necrosis, inhomogeneous enhancement, and a plurality of nodes integration. The planning gross tumor volume $\left(\mathrm{PTV}_{\mathrm{G}}\right)$ was established uniformly by including a $5 \mathrm{~mm}$ margin around the GTV only in SIR-IMRT group. Clinical target volume (CTV) was defined as the high-risk lymph nodal regions, including adjacent regions of involved lymph nodes and the ipsilateral hila and the GTV with a $5 \mathrm{~mm}$ margin. The $5 \mathrm{~mm}-10 \mathrm{~mm}$ expansion to CTV was defined as the planning target volume (PTV). In SIR-IMRT group, the prescribed radiation dose was 60Gy to $\mathrm{PTV}_{\mathrm{G}}$ with a division of 2.0Gy/day and 54Gy to the PTV with a division of 1.8Gy/day, 5 fractions per week. In C-IMRT group, the prescribed radiation dose for PTV was 60Gy, 2.0Gy/day. The dose deliveries were $\geq 95 \%$ for all the target volumes. Each treatment plan consisted of five static fields with the following normal tissue constraints: 1) total lung, $\mathrm{V}_{\text {lung }} 5$ (i.e., the percentage of lung volume receiving $\geq$ 5 Gy) $\leq 60 \%$ and $V_{\text {lung }} 20 \leq 35 \%$; 2) $V_{\text {lung }} 40 \leq 30 \%$; 3) $\mathrm{V}_{\text {esophagus }} 50 \leq 50 \%, \mathrm{~V}_{\text {esophagus }}$ maximum $\leq 60 \mathrm{~Gy}$; and 4 ) $\mathrm{V}_{\text {spinal cord }}$ maximum $\leq 45 \mathrm{~Gy}$. The relation of dose and volume of the target volume and OAR was evaluated by the dose volume histogram (DVH). For patients who achieved a complete response (CR) after thoracic radiotherapy, prophylactic cranial irradiation (PCI) was recommended, with a dose of 25Gy administered over 10 fractions.

Etoposide and cisplatin or carboplatin were combination in chemotherapy, the dose of the etoposide was $60 \mathrm{mg} / \mathrm{m}^{2}$ (intravenous administration for 5 days) and cisplatin $30 \mathrm{mg} / \mathrm{m}^{2}$ (intravenous 
administration for 3 days) or carboplatin AUC $=6$ at first day. The treatment schedule of all patients was shown in Table 2.

Table 2. The presentation of the patient receiving treatment $(n=$ 84)

\begin{tabular}{|c|c|c|c|}
\hline Characteristics & $\begin{array}{l}\text { SIR-IMRT } \\
\text { No.(\%) }\end{array}$ & C-IMRT No.(\%) & $P$ \\
\hline \multicolumn{4}{|c|}{ Induction chemotherapy } \\
\hline Yes & $42(100.0 \%)$ & $42(100.0 \%)$ & 1.0 \\
\hline No & 0 & 0 & \\
\hline \multicolumn{4}{|c|}{$\begin{array}{l}\text { Cycles of Induction } \\
\text { chemotherapy }\end{array}$} \\
\hline$<3$ & $21(50.0 \%)$ & $29(69.0 \%)$ & 0.108 \\
\hline$\geq 3$ & $21(50.0 \%)$ & $13(31.0 \%)$ & \\
\hline \multicolumn{4}{|c|}{$\begin{array}{l}\text { Response post Induction } \\
\text { chemotherapy }\end{array}$} \\
\hline $\mathrm{CR}+\mathrm{PR}$ & $27(64.3 \%)$ & $29(69.0 \%)$ & 0.817 \\
\hline $\mathrm{SD}+\mathrm{PD}$ & $15(35.7 \%)$ & $13(31.0 \%)$ & \\
\hline \multicolumn{4}{|c|}{ Adjuvant chemotherapy } \\
\hline Yes & $23(54.8 \%)$ & $26(61.9 \%)$ & 0.658 \\
\hline No & $19(45.2 \%)$ & $16(38.1 \%)$ & \\
\hline \multicolumn{4}{|c|}{$\begin{array}{l}\text { Concurrent radiation } \\
\text { with chemotherapy }\end{array}$} \\
\hline Yes & $15(35.7 \%)$ & $18(42.9 \%)$ & 0.655 \\
\hline No & $27(64.3 \%)$ & $24(57.1 \%)$ & \\
\hline \multicolumn{4}{|c|}{$\begin{array}{l}\text { Prophylactic cranial } \\
\text { irradiation }\end{array}$} \\
\hline Yes & $18(42.9 \%)$ & $20(47.6 \%)$ & 0.512 \\
\hline No & $24(57.1 \%)$ & $22(52.4 \%)$ & \\
\hline
\end{tabular}

Follow up All patients were followed up to December 31, 2015 from the completion of the radiation treatment. The median follow-up period was 40.2 months. Patients were followed up at the $1^{\text {st }}$ and $3^{\text {rd }}$ month after radiotherapy, then, once every three months for 2 years, and once every six months thereafter. Examinations included neck and abdomen ultrasound, chest CT scan, cranial CT or MR and bone scan if necessary.

Response and Toxicity The radiation-related toxicities of the lung and esophageal were evaluated by two senior radiation therapists according to the Common Terminology Criteria for Adverse Events (CTCAE) Version 3.0. The response was conducted 3 months after the radiotherapy based on new guidelines designed to evaluate treatment response by solid tumors. The response was divided into complete response (CR), partial response (PR), stable disease (SD) and progress disease (PD) 10. Loco-regional recurrence (LRR) was divided into local recurrence and regional recurrence: The local recurrence was defined as recurrence within the $95 \%$ isodose curve of GTV $\left(\mathrm{PTV}_{\mathrm{G}}\right)$. The regional recurrence field was defined as out of the $95 \%$ isodose curve of GTV target area, but still confined to the lung, pulmonary, mediastinal and supraclavicular regions without distant metastasis.

Statistical analysis Loco-regional recurrencefree survival (LRFS), Progression-free survival (PFS), distant metastases-free survival (DMFS), and overall survival (OS) were estimated with the Kaplan-Meier method. The endpoint for OS was the date of death or the date of last follow-up. The endpoint for LRFS was the recurrence of the primary tumor or regional lymph node or the date of last follow-up. The endpoint for DMFS was the date of distant metastases developed at first. The endpoint for PFS was the date disease progression was detected or the last follow-up date. The statistical analysis was performed with SPSS17.0 software.

\section{Results}

Normal tissue dosimetry parameters All of patients completed thoracic radiotherapy. It can be illustrated that the delivered dose of OARs in SIR-IMRT group were lower than the OARs in the C-IMRT group, but there was no statistically significance. The dose parameters of the OARs of the two groups were shown in Table 3.

Table 3. The dose volume parameter of organ at risk (OAR) $(\mathrm{n}=84)$

\begin{tabular}{|c|c|c|c|}
\hline OAR & SIR-IMRT & C-IMRT & $P$ \\
\hline $\begin{array}{l}\text { Mean lung dose (MLD) } \\
\text { (cGy) }\end{array}$ & $1494 \pm 161.51$ & $1515 \pm 136.56$ & 0.470 \\
\hline Vlung5 (\%) & $48.02 \pm 6.11$ & $49.52 \pm 7.19$ & 0.227 \\
\hline$V_{\text {lung } 20(\%)}$ & $27.52 \pm 2.57$ & $28.57 \pm 3.43$ & 0.153 \\
\hline$V_{\text {lung } 30(\%)}$ & $20.17 \pm 2.89$ & $20.19 \pm 2.48$ & 0.731 \\
\hline EsophagusD $_{\max }(\mathrm{cGy})$ & $6112 \pm 304$ & $6365 \pm 230$ & 0.301 \\
\hline $\mathrm{V}_{\text {esophagus }} 50(\%)$ & $28.31 \pm 11.46$ & $29.19 \pm 14.32$ & 0.086 \\
\hline Cord $D_{\max }(\mathrm{cGy})$ & $4447 \pm 345$ & $4452 \pm 307$ & 0.479 \\
\hline
\end{tabular}

Survival analysis Three months after the radiotherapy, the overall response $(\mathrm{CR}+\mathrm{PR})$ rates of patients was 95.2\% (40/42) in SIR-IMRT group and 92.9\% (39/42) in C-IMRT group, respectively. The median survival time of all patients was 25.5 months. The 1- ,2-, 3-, and 5-year LRFS rates of the SIR-IMRT group and C-IMRT group were $90.4 \%, 76.8 \%, 73.3 \%$, $73.3 \%$ and $87.1 \%, 61.7 \%, 45.0 \%, 15.0 \% \quad(P=0.025)$, respectively, while the 1- ,2-, 3- and 5- year OS rates were $90.5 \%, 62.0 \%, 48.8 \%, 48.8 \%$ and $83.3 \%, 45.2 \%$, $38.1 \%, 16.9 \%(P=0.037)$, respectively. The $1-, 2-, 3-$, and 5- year PFS rates were $68.4 \%, 44.0 \%, 39.1 \%, 39.1 \%$ and $63.0 \%, 29.7 \%, 20.8 \%, 6.9 \%(P=0.065)$, respectively. The 1- ,2-, 3-, and 5- year DMFS rates were 72.9\%, 51.4\%, $48.6 \%, 48.6 \%$ and $65.1 \%, 36.0 \%, 30.9 \%, 30.9 \%$ $(P=0.270)$, respectively. (Figure 1$)$.

Patterns of failure By the last follow-up, 58 cases $(69.0 \%)$ of patients experienced treatment failure, of which, LRR developed in 27 patients $(32.1 \%)$, and distant metastases were detected in 45 patients $(53.6 \%)$. Fifteen patients experienced both loco-regional and distant failure. In SIR-IMRT group, 
A

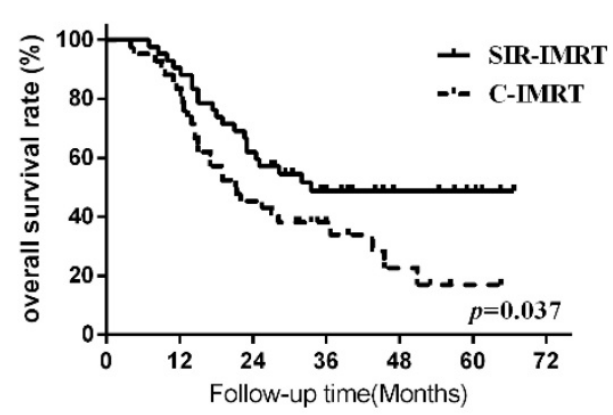

C

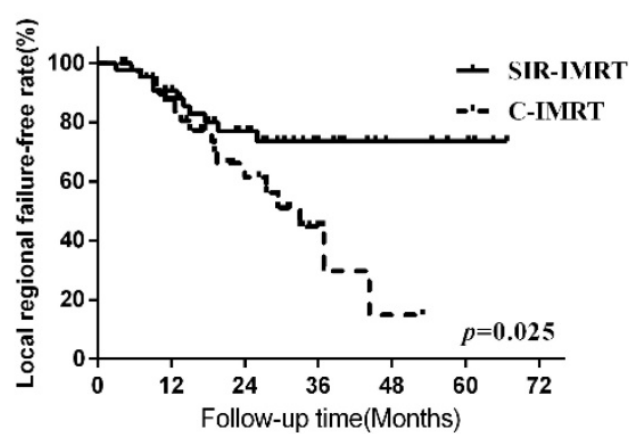

B

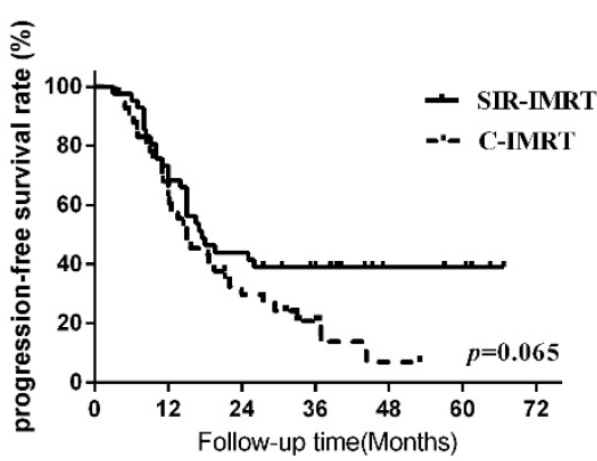

D

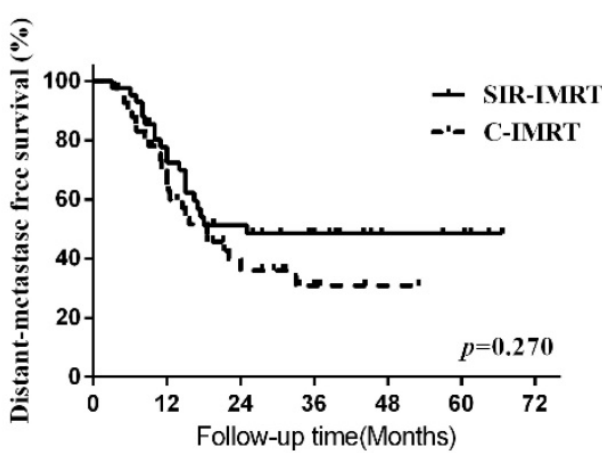

Figure 1. Survival Curve

10 patients experienced LRR (7 cases in the GTV, 3cases in the regional field), while in C-IMRT group, 17 patients experienced LRR ( 13 cases in the GTV, 4 cases in the regional field). The analysis of the reasons for the failures was shown in Table 4.

Table 4. Patterns of failure for recurrence $(n=84)$

\begin{tabular}{lll}
\hline Recurrence & SIR-IMRT No. & C-IMRT No. \\
\hline Loco-regional recurrence & 10 & 17 \\
Local(GTV) & 7 & 13 \\
Regional & 3 & 4 \\
Distant metastasis & 20 & 25 \\
Bone & 3 & 6 \\
Liver & 6 & 6 \\
Celiac lymph nodes & 2 & 3 \\
Brain & 12 & 12 \\
Adrenal gland & 1 & 5 \\
Heart & 1 & 0 \\
Pancreas & 0 & 2 \\
Loco-regional recurrence & 5 & 10 \\
and distant metastasis & & \\
\hline
\end{tabular}

Table 5. Treatment-related toxicity $(n=84)$

\begin{tabular}{lll}
\hline & \multicolumn{1}{l}{ SIR-IMRT No.(\%) } & C-IMRT No.(\%) \\
\hline Lung & & \\
Grade 0-1 & $30(71.4 \%)$ & $23(54.8 \%)$ \\
Grade 2 & $10(23.8 \%)$ & $14(33.3 \%)$ \\
Grade 3 & $1(2.4 \%)$ & $3(7.1 \%)$ \\
Grade 4 & $0(0.0 \%)$ & $0(0.0 \%)$ \\
Grade 5 & $1(2.4 \%)$ & $2(4.8 \%)$ \\
Esophagus & & \\
Grade 0-1 & $22(52.4 \%)$ & $17(40.5 \%)$ \\
Grade 2 & $17(40.5 \%)$ & $20(47.6 \%)$ \\
Grade 3 & $3(4.8 \%)$ & $5(11.9 \%)$ \\
Grade 4 & $0(0.0 \%)$ & $0(0.0 \%)$ \\
Grade 5 & $0(0.0 \%)$ & $0(0.0 \%)$ \\
\hline
\end{tabular}

Treatment-related Toxicity In both of the two groups, we observed various grades of treatment-related lung toxicity and treatment-related esophagitis. For example, there were 2 patients in the SIR-IMRT group and 5 patients in the C-IMRT group experienced treatment-related pneumonia (TRP) above grade 3, respectively. Grade 5 TRP happened in 1 patient in the SIR-IMRT group and 2 patients in the C-IMRT group respectively. 3 patients in the SIR-IMRT group and 5 patients in the C-IMRT group experienced treatment-related esophagitis above grade 3, respectively. The treatment-related toxicity and adverse reactions of the two groups were illustrated in Table 5.

\section{Discussion}

The encouraging results of this study indicated that SIR-IMRT reduced toxicity of OARs without reducing LRFS, OS and PFS compared to C-IMRT for patients with LS-SCLC. Shalini Garg's ${ }^{11}$, Rosenzweig KE's ${ }^{12}$ and others' studies showed that in NSCLC, selective regional recurrence was extremely rare, most of LRR still occurred in the radiation field. So the involved field radiation in lymphatic drainage area was suggested to reduce the radiation area of the CTV and decrease the toxicity of the OAR, with no affect on the survival of patients. There wasn't such study reported in the LS-SCLC treatment yet. This study applied the involved field radiation, and the result showed that local recurrence occurred in the radiation 
field except one out of field. In SIR-IMRT group, regional recurrence in supraclavicular area occurred in 3 patients. These patients were all confirmed to be metastatic of supraclavicular lymph nodes before chemotherapy and the response of the chemotherapy was almost CR. So we gave the prophylactic dose to the supraclavicular metastatic lymph nodes. Unfortunately, recurrence still occurred in these areas. In C-IMRT group, failure in the marginal of the irradiation field occurred in 1 patient, and failure in the CTV occurred in 3 patients. The local control rate of the SIR-IMRT group wasn't lower than that of the C-IMRT group, even when the radiation dose to the CTV in SIR-IMRT group was reduced. 1- and 3- year LRFS rates were $90.4 \%$ and $73.3 \%$ for the SIR-IMRT group, while $87.1 \%$ and $45.0 \%$ for the C-IMRT group, $(P=0.025)$. The result also showed that the local recurrence mainly occurred in the GTV and regional recurrences were common in the preliminarily diagnosed positive lymph nodes areas.

In this study, two- year OS and PFS rates were $62.0 \%$ and $44.0 \%$ in SIR-IMRT group, while $45.2 \%$ and $29.7 \%$ in C-IMRT group, respectively. A phase II study (RTOG0239) ${ }^{13}$ investigated the efficacy and feasibility of accelerated radiotherapy involving a total dose of 61.2Gy concurrent with chemotherapy for SCLC. The median OS and PFS were 19.0 months and 9.9 months, respectively. The 2- year OS rate PFS rate was $36.6 \%$ and $19.7 \%$, the results were disappointed. However, this study achieved better OS and PFS than the RTOG0239 study, even with lower prescribed radiation dose. In Shirvani, et al's study ${ }^{14}$, the median dose of PTV was 45Gy/30f (40.5Gy/ 27f-63.8Gy/35f) with PET-CT guided IMRT in LS-SCLC, 2- year OS and PFS rate was 58\% and $43 \%$, which were approximate to our results. The results of the CONVERT trials with the purpose to compare the differences hypo radiotherapy (45Gy, 1.5Gy/f bid) to conventional radiotherapy (66Gy, 2Gy/f qd) were reported on ASCO in 2016. The median follow-up time was 45 months, 2- years survival rate was $56 \%$ and $51 \%$ and the median survival was 30 months and 25 months $^{15}$, respectively. We can find that in the above studies, although the PTV was given a higher dose, OS and PFS were not proved to be better. Our study showed that in SIR-IMRT, the reduction of the dose for CTV didn't reduce the OS and PFS.

The results about toxicity reported in the present study were also encouraging. The rate of grade 3 esophagitis was $7.1 \%$ and $11.9 \%$ in SIB-IMRT group and in C-IMRT, respectively. While the rate of TRP of grade 3 or greater was $4.8 \%$ and $11.9 \%$, respectively. A recent report showed that the incidences of radiation related esophagitis and TRP (grade 3 ) were between $18-23 \%$ and $7-11 \% 13,14$ in non-small cell lung cancer or
SCLC treated with IMRT, respectively. In this study, the toxicities of OARs in SIB-IMRT group were significantly lower than that of C-IMRT group and previously published dates, especially lung toxicity. The use of the SIR-IMRT technique also resulted in the application of a dose gradient to the $\mathrm{PTV}_{\mathrm{G}}$ and PTV. It had the benefit of guarantee for the treatment dose of tumor and meanwhile the protection for proximal normal organs especially for lung V20. Correspondingly, in a recent meta-analysis, symptomatic pneumonitis increased by $3 \%$ when lung $\mathrm{V}_{20}$ increased by $1 \%{ }^{16}$. The lung V20 of two groups in this study were $27.52 \pm 2.57$ and $28.57 \pm 3.43$ (\%), respectively, it might be associated with high incidence of TRP above grade-3 in C-IMRT group.

The results of this study concluded that compared to C-IMRT group, reducing the dose of the CTV didn't increase the rate of the local recurrence in SIB-IMRT group, but reduced the toxicity of the OARs. The survival results of the two groups showed that local recurrence mostly located in GTV. Therefore, we believe that SIB-IMRT therapy is safe and feasible for LS-SCLC. We will carry out further research on the recurrence of the GTV, in hope of better LRFS and OS by increasing GTV dose, while reducing the prophylactic irradiation to CTV and the radiation dose of the OARs.

\section{Conclusion}

There are several limitations in this study: this is a retrospective research, so selection bias might result underestimates of tumor recurrence and death rate, although we had balanced the dates of the two groups by propensity score matching. The encouraging results of this study indicated that SIR-IMRT reduced toxicity of OARs without reducing LRFS, OS and PFS for patients with LS-SCLC compared to C-IMRT, and also provided an intriguing justification for future. According to the results of this study, our next work is to research on reducing the irradiation dose to the elective nodal region and increasing the $\mathrm{PTV}_{\mathrm{G}}$ dose for LS-SCLC.

\section{Acknowledgments}

This work was supported by grants from the National Natural Science Foundation of China (Grant No. 81502659 and 81201753 ).

\section{Competing Interests}

The authors have declared that no competing interest exists.

\section{References}

1. Pignon JP, Arriagada R, Ihde DC, et al. A meta-analysis of thoracic radiotherapy for small-cell lung cancer. N Engl J Med. 1992; 327:1618-24. 
2. Warde P, Payne D. Does thoracic irradiation improve survival and local control in limited-stage small-cell carcinoma of the lung? A meta-analysis. J Clin Oncol. 1992; 10:890-5.

3. Hann CL, Rudin CM. Management of small-cell lung cancer: incremental changes but hope for the future. Oncology (Williston Park). 2008; 22: 1486-92.

4. Schild SE, Bonner JA, Shanahan TG, et al. Long-term results of a phase III trial comparing once-daily radiotherapy with twice-daily radiotherapy in limited-stage small-cell lung cancer. Int J Radiat Oncol Biol Phys. 2004; 59: 943-51.

5. Komaki R, Swann RS, Ettinger DS, et al. Phase I study of thoracic radiation dose escalation with concurrent chemotherapy for patients with limited small-cell lung cancer: Report of Radiation Therapy Oncology Group (RTOG) protocol 97-12. Int J Radiat Oncol Biol Phys. 2005; 62: 342-50.

6. Schild SE, Bonner JA, Hillman S, et al. Results of a phase II study of high-dose thoracic radiation therapy with concurrent cisplatin and etoposide in limited-stage small-cell lung cancer (NCCTG 95-20-53). J Clin Oncol. 2007; 25: 3124-9.

7. Aridgides PD, Movsas B, Bogart JA. Thoracic radiotherapy for limited stage small cell lung carcinoma. Curr Probl Cancer. 2012; 36: 88-105.

8. Bradley JD, Paulus R, Komaki R, et al. Standard-dose versus high-dose conformal radiotherapy with concurrent and consolidation carboplatin plus paclitaxel with or without cetuximab for patients with stage IIIA or IIIB non-small-cell lung cancer (RTOG 0617): a randomised, two-by-two factorial phase 3 study. Lancet Oncol. 2015; 16:187-99.

9. Withers HR, Peters LJ, Taylor JM. Dose-response relationship for radiation therapy of subclinical disease. Int J Radiat Oncol Biol Phys. 1995; 31:353-9.

10. Eisenhauer EA, Therasse P, Bogaerts J, et al. New response evaluation criteria in solid tumours: revised RECIST guideline (version 1.1). Eur J Cancer. 2009; 45:228-47.

11. Garg S, Gielda BT, Kiel K, et al. Patterns of locoregional failure in stage III non-small cell lung cancer treated with definitive chemoradiation therapy. Pract Radiat Oncol. 2014; 4:342-8.

12. Rosenzweig KE, Sura $S$, Jackson A, et al. Involved-field radiation therapy for inoperable non small-cell lung cancer. J Clin Oncol. 2007; 25:5557-61.

13. Komaki R, Paulus R, Ettinger DS, et al. Phase II study of accelerated high-dose radiotherapy with concurrent chemotherapy for patients with limited small-cell lung cancer: Radiation Therapy Oncology Group protocol 0239. Int J Radiat Oncol Biol Phys. 2012; 83: e531-6.

14. Shirvani SM, Komaki R, Heymach JV, et al. Positron emission tomography/computed tomography-guided intensity-modulated radiotherapy for limited-stage small-cell lung cancer. Int J Radiat Oncol Biol Phys. 2012; 82: e91-7.

15. Faivre-Finn C, Michael Snee, Ashcroft L, et al. CONVERT: An international randomised trial of concurrent chemoradiotherapy(cCTRT) comparing twice-daily(BD) and once-daily(QD) radiotherapy schedules in patients with limited stage small cell lung cancer (LS-SCLC) and good performance status(PS). Lung Cancer. 2017; 103: S64-4.

16. Palma DA, Senan S, Oberije $C$, et al. Predicting radiation pneumonitis after chemoradiation therapy for lung cancer: an international individual patient data meta-analysis. Int J Radiat Oncol Biol Phys. 2013; 85:444-50. 